Revue d'histoire de l'Amérique française

RAS REVUE D.HISTOIRE DE L'AMÉRIQUE FRANÇAISE

\title{
Race et religion, les Acadiens et la hiérarchie catholique irlandaise du Nouveau-Brunswick
}

\section{Martin S. Spigelman}

Volume 29, numéro 1, juin 1975

URI : https://id.erudit.org/iderudit/303418ar

DOI : https://doi.org/10.7202/303418ar

Aller au sommaire du numéro

Éditeur(s)

Institut d'histoire de l'Amérique française

ISSN

0035-2357 (imprimé)

1492-1383 (numérique)

Découvrir la revue

Citer cet article

Spigelman, M. S. (1975). Race et religion, les Acadiens et la hiérarchie catholique irlandaise du Nouveau-Brunswick. Revue d'histoire de l'Amérique française, 29(1), 69-85. https://doi.org/10.7202/303418ar d'utilisation que vous pouvez consulter en ligne. 


\title{
RACE ET RELIGION \\ LES ACADIENS ET LA HIÉRARCHIE CATHOLIQUE IRLANDAISE DU NOUVEAU-BRUNSWICK
}

\author{
Martin S. Spigelman \\ Département d'histoire \\ Université de Dalhousie \\ Halifax, Nouvelle-Ecosse
}

Interrogé, deux ans avant sa mort, en 1933, sur les luttes des Acadiens qu'il avait personnellement menées durant près d'un demi-siècle, Pascal Poirier ${ }^{1}$ donna une réponse qui traduisait bien l'âme acadienne, toute conciliante et dépourvue d'agressivité:

Nous avons gagné l'essentiel - l'égalité dans la hiérarchie, la paix religieuse avec le clergé irlandais; l'estime de nos concitoyens anglais; la tolérance de l'enseignement du français. Il est préférable, je crois, de ne pas remuer le passé - let well enough alone. ${ }^{2}$

Cependant, il s'étendit volontiers sur le conflit qui, durant quarante ans, opposa les Acadiens catholiques du NouveauBrunswick à la hiérarchie et au clergé catholiques irlandais. Durant la première partie du XIXème siècle, les Acadiens étaient réduits au désespoir même s'ils semblaient peu affectés par leur triste situation. En effet, ils se montraient peu intéressés à protéger une culture et une langue qu'ils avaient pourtant réussi à préserver dans un passé encore plus défavorable. Mais, à l'époque de la Confédération et durant la dernière partie du XIXème siècle, stimulés par une élite instruite, ambitieuse et énergique, ils réagirent peu à peu à leur position défavorable

1 Pascal Poirier (1852-1933) naquit à Shediac (N.-B.) et il fit ses études au Collège Saint-Joseph de Memramcook. Il fut directeur général des postes au Parlement en 1867 et, en 1885, il devint le premier Acadien à accéder au sénat. Avocat autodidacte, écrivain prolifique, minéralogiste passionné, membre de la Société royale du Canada et chevalier de la Légion d'honneur de France, Poirier fut le premier leader de la Renaissance acadienne.

2 Le sénateur Pascal Poirier au Père Albert Poirier, 10 mars 1931, Centre d'Etudes acadiennes, Moncton (ci-après CEA), Coll. Poirier, 6, 4-1. Le souligné est de l'auteur. 
au sein des provinces maritimes. ${ }^{3}$ Leurs dirigeants pouvaient et voulaient saisir les avantages que leur offrait la Confédération et, de fait, ils réussirent remarquablement sur la scène politique. Toutefois, dans l'Eglise, il en fut tout autrement, l'accès aux rangs supérieurs leur étant strictement refusé par une hiérarchie catholique irlandaise qui s'y retranchait. En conséquence, au tournant du siècle, le combat des Acadiens pour la conservation d'une identité culturelle distincte était mené davantage contre les Irlandais que contre les Anglais.

$\mathrm{Au}$ début du XIXème siècle, la position des Acadiens était moins qu'enviable. En visite chez eux, Monseigneur Plessis, de Québec, disait: "Il n'y a peut-être pas de peuple dans tout le Canada où l'on voit autant de mésintelligence, de querelles et de haines réciproques." Ils étaient "en général assez mauvais cultivateurs", constamment endettés et asservis aux magnats locaux de l'industrie du bois et des pêcheries ${ }^{4}$. Il n'y avait, pour ainsi dire, pas de moyens d'éducation; d'ailleurs, le peuple acadien s'en souciait peu ${ }^{5}$. La langue qu'ils avaient conservée à travers un siècle de tribulations s'était corrompue d'anglicismes et était menacée d'extinction ${ }^{6}$. Le clergé, aussi bien irlandais qu'écossais ou canadien-français, s'intéressait davantage à la dîme qu'au bien-être des fidèles 7. Les communautés acadiennes, disséminées dans le nord du Nouveau-Brunswick, au Cap-Breton, sur la rive sud de la Nouvelle-Ecosse, dans l'Ile du Prince-Edouard étaient non seulement isolés les unes des autres, mais aussi, contrairement aux communautés irlandaises, éloignées des villes où s'exerçait l'autorité politique et ecclésiastique.

3 Dans le présent article, le mot "Acadien" désigne les 80,000 francocatholiques du Nouveau-Brunswick (Recensement du Canada, 1901, 290) qui, en fait, avaient des intérêts et des aspirations en commun. Cependant, c'est la partie sud de la province qui donne l'impulsion à la Renaissance acadienne et lui fournit ses leaders. Les comtés de Madawaska et de Victoria, au nord, furent moins intéressés par ce mouvement parce qu'ils subissaient davantage l'influence du Québec.

4 Journal de Mgr J.-O. Plessis écrit durant sa visite pastorale de 1811 en Acadie. Voir "Journal de Mgr J.-O. Plessis", dans L'Ordre Social (Moncton), 9 janv. - 24 juillet 1938. Les numéros 7, 11 et 13 de cette série sont particulièrement intéressants.

5 Voir le Moniteur acadien (Shediac), 18 juillet 1867 , ou L'Evangéline (Weymouth, N.-E.), 12 déc. 1895.

Rameau, 2.1-2.

6 Abbé Blanchet à Rameau de Saint-Père, 17 août 1860, CEA, Coll.

7 Voir la copie du discours prononcé par Rameau de Saint-Père à Québec le 23 oct. 1860, Coll. Rameau, CEA, 2.5-2. Voir aussi Pierre-A. Landry, "Les Acadiens" (manuscrit du 24 août 1901), CEA, Coll. Landry, 4.4-11. 
Les Acadiens acceptaient avec résignation la pauvreté et le mépris dont ils étaient l'objet. Le passé leur avait appris à se satisfaire de peu. Ils n'aspiraient, d'après Poirier, qu'à vivre en paix avec le peu qu'ils possédaient et ne demandaient qu'à être ignorés ${ }^{8}$. Ils acceptaient inconditionnellement leur exclusion de la société et de la hiérarchie de l'Eglise, comme la conséquence de leur manque d'éducation; ils languissaient dans la pauvreté intellectuelle et la misère matérielle, développant collectivement un profond complexe d'infériorité. La soumission à la majorité anglophone était devenue une seconde nature:

We have become so accustomed to this idea of inferiority that ... we consider it necessary to surround the English with a deference that we might quite properly accord to our own countrymen. If an Englishman settled in the midst of a thoroughly French district... he could at once by general consent become the ruling spirit of the district ... And if one of our own found his way into an English district, it was to become the servant, the menial of the English. ${ }^{9}$

En conséquence, dans les cinq diocèses des provinces maritimes ${ }^{10}$, les Irlandais et les Ecossais s'emparèrent des évêchés, et leur domination ne fut jamais contestée jusqu'en 1900, alors que les évêques John Sweeny (Saint John) et James Rogers (Chatham) atteignirent l'âge de la retraite.

Les Acadiens, passifs jusqu'alors, changèrent radicalement d'attitude dans les dernières décades du XIXème siècle. Tout d'abord, leur population s'accrut, donnant plus de poids à leur réclamation d'une représentation plus adéquate. Dès 1901, en effet, les catholiques d'expression française dépassaient en nombre les catholiques irlandais, dans une proportion voisine de deux pour un ${ }^{11}$. De plus, les nouveaux collèges au service des paroisses acadiennes et les Conventions Nationales qui se multipliaient avaient formé une élite, dont Pascal Poirier, le Père Marcel

8 Pascal Poirier, discours prononcé à Montréal en juin 1884, réimprimé dans F.-J. Robidoux, Conventions nationales des Acadiens (Shediac, 1907), 192-193.

9 P.-A. Landry, cité dans le Daily Times (Moncton), coupure de journal sans date conservée au CEA, Coll. Landry, 5.4-1.

10 Les cinq diocèses sont Halifax (fondé en 1818), Charlottetown (1829), Arichat (1844), New Brunswick (1842) et Chatham (1860).

11 Voir Canada, Ministère de l'agriculture, Fourth Census of Canada, 1901 (Ottawa, 1902), I: 160, 290. Il y avait 80,000 citoyens d'origine française, 83,000 d'origine irlandaise et 48,000 d'origine écossaise au NouveauBrunswick. Cependant, il n'y avait que 126,000 catholiques romains. On peut supposer, sans crainte de se tromper, que la grande majorité des francophones était catholique alors que seulement la moitié des Irlandais l'était. 
Richard et Pierre-A. Landry. Convaincue de la justesse de la cause qu'elle défendait, cette élite pouvait presser le peuple acadien d'exiger ce qui lui revenait en justice. Comprenant surtout des laïcs, elle remplit un rôle important. Respectée par l'ensemble de la société acadienne, cette élite témoigna d'un réel sens social et assuma ses responsabilités à l'égard des paroisses et du peuple ${ }^{12}$. Elle sut saisir les occasions favorables, définir les objectifs à atteindre et encourager le peuple dans ses efforts d'émancipation.

En 1900, la société acadienne était en pleine effervescence. Sortis de leur analphabétisme et parvenus à une meilleure situation sociale, les Acadiens se montraient fiers du nombre de leurs médecins et de leurs avocats, de leurs trois périodiques d'expression française et de leur banque française dirigée par un Acadien ${ }^{13}$. Ils avaient fondé une association nationale, La Société nationale l'Assomption, ainsi qu'une société de crédit purement acadienne, La Société mutuelle l'Assomption. La langue française qui constituait autrefois "une source de faiblesse, sinon de honte", devenait une force ${ }^{14}$. On trouvait maintenant des Acadiens à la fonction publique, à la Chambre des Communes, au Sénat, aux Assemblées des trois provinces maritimes et aux tribunaux du Nouveau-Brunswick. Le peuple acadien avait fait des progrès substantiels dans le domaine séculier, parce qu'il avait repris confiance en lui-même et refait son unité.

Toutefois, une institution restait encore absolument fermée aux Acadiens. C'était leur propre Eglise. "Quelle différence, s'exclamait un des leurs, entre notre influence temporelle sans cesse grandissante, et notre influence religieuse, nulle!" ${ }^{15}$. On comprend que Pascal Poirier ait affirmé avec amertume: "Les anglais protestants sont mieux disposés à nous accorder nos droits, que nos coreligionnaires irlandais." 16 Le fait que les Acadiens étaient en majorité dans l'Eglise s'avérait de peu d'importance contre la puissance d'une hiérarchie anglophone, solidement établie et déterminée à perpétuer sa domination.

$12 \mathrm{Au}$ sujet des élites dans la société acadienne ou canadienne-française voir M.-A. Tremblay, "Les Acadiens de la Baie Française, l'histoire d'une Survivance", Revue d'histoire de l'A mérique française, 15, no 4 (mars 1962) : 526-55; L'Evangéline, 2 avril, 1913; Le Devoir (Montréal), 1er juillet 1914. 13 L'Evangéline, 7 sept. 1910.

14 Le Moniteur acadien, 30 déc. 1896. dry, 7.2-10.

15 Valentin Landry, manuscrit inédit, mai 1911, CEA, Coll. V.-A. Lan2.1-31.

16 P. Poirier à Rameau de Saint-Père, 10 avril 1892, CEA, Coll. Rameau, 
La préoccupation première des Acadiens entre 1890 et 1912 fut de lutter pour l'égalité dans le domaine religieux. Il leur fallait sans aucun doute un évêque acadien qui pût comprendre les aspirations de son propre peuple et les efforts de son clergé. $\mathrm{Au}$ contraire, la hiérarchie irlandaise prétendait que, dans le choix d'un évêque, la nationalité était hors de cause, que la foi seule comptait. Si le rôle de l'Eglise s'était limité au seul domaine spirituel, ce principe aurait été acceptable mais, dans les paroisses françaises en particulier, l'Eglise avait aussi une fonction temporelle. Le curé de la paroisse était plus qu'un "homme de sacristie". C'était le conseiller en qui on mettait sa confiance, l'arbitre des querelles à l'intérieur de la paroisse et l'intermédiaire entre les paroissiens et le gouvernement. Parfois même, le prêtre n'hésitait pas à se mêler de politique, si la cause justifiait son intervention. Il jouait un rôle important dans la colonisation, dans le mouvement de tempérance, dans l'éducation et, en fait, en toutes choses, parce que c'était là "précisément la tâche du pasteur, le devoir du prêtre" "17. Tous ses fidèles, jeunes et vieux, le nommaient "Père", et cette appellation correspondait exactement à son rôle.

Cependant, selon le témoignage de plusieurs Acadiens, les prélats catholiques irlandais du Nouveau-Brunswick se servaient du pouvoir de l'Eglise pour entraver le progrès de la population acadienne. Dès 1863, un prêtre français de Bouctouche, le Père H.-L. Berthe, craignait l'extinction de la race acadienne du fait que le haut-clergé était entièrement irlandais. Il s'appuyait sur l'expérience d'un prêtre belge de la Nouvelle-Ecosse, l'abbé Warlop, qui voulut porter à Rome les doléances acadiennes. Un mot en parvint à l'archevêque d'Halifax; l'abbé Warlop fut interdit et dut chercher refuge à Trinidad, aux Antilles ${ }^{18}$. Même s'ils étaient à court de prêtres d'expression française dont ils avaient grand besoin, les évêques refusèrent souvent de recevoir du Québec un missionnaire francophone, sous prétexte qu'il ne maîtrisait pas la langue anglaise. On vit même des Acadiens qui voulaient se confesser dans leur langue adresser à Mgr Sweeny une requête à cet effet. Celui-ci répliqua en les accusant d'infidélité à l'Eglise, et quelques prêtres irlandais les menacèrent même d'excommunication ${ }^{19}$. La Congrégation des Petites Sœurs de la

17 Le Moniteur acadien, 20 sept. 1895.

18 Abbé H.-L. Berthe à Rameau de Saint-Père, 12 avril 1863, Coll., Rameau, 2.1-5. Le souligné est de l'auteur.

19 Abbé H.-L. Berthe à Rameau de Saint-Père, 5 mai 1864, CEA, Coll., Rameau, 2.1-6. 
Sainte-Famille, très appréciée des Acadiens, se vit refuser la reconnaissance canonique par Mgr Sweeny et dut s'adresser à Mgr Paul LaRoque, de Sherbrooke (Québec) ${ }^{20}$. Même au sein des différents ordres religieux, il y avait de profondes rivalités entre les membres acadiens et irlandais. L'épiscopat, pour sa part, encourageait les catholiques irlandais à devenir prêtres et décourageait les aspirants acadiens. Lorsque l'élite acadienne voulut fonder des journaux pour renseigner et éduquer la population, les évêques, d'un commun accord, s'objectèrent à ce projet, sous prétexte qu'il était superflu d'éditer des journaux français, alors que les journaux anglais remplissaient déjà cette fonction ${ }^{21}$. La liste des conflits, mesquins ou sérieux, serait inépuisable.

Les évêques catholiques des provinces maritimes attachaient beaucoup d'importance à un système d'écoles confessionnelles et, avant la Confédération, ils travaillèrent d'arrache-pied pour obtenir les mêmes droits scolaires que les minorités des deux Canadas. Dans la lutte inutile qu'il mena à cette fin, Mgr Connolly, d'Halifax, alla jusqu'à faire cette promesse "that neither (Joseph) Howe or any of his kind will succeed ... in any Catholic constituency in either of the Maritime Provinces" ${ }^{22}$. Les Acadiens réclamaient aussi le système des "écoles séparées". Mais c'était le statut de la langue française dans ces écoles qui leur tenait le plus à cœur, ce qui n'était évidemment pas une des priorités de Mgr Connolly, même si son diocèse était au tiers acadien ${ }^{23}$. Nulle part dans sa correspondance avec les chefs politiques, il n'est question du statut de la langue française dans les "écoles séparées". L'inquiétude des Acadiens à ce sujet semblait échapper à ses préoccupations et à sa compréhension.

La loi du Parlement provincial de Frédéricton qui, en mai 1871, retirait toute subvention aux écoles catholiques, affecta plus les catholiques acadiens qu'irlandais. Les Acadiens venaient tout juste de prendre conscience de la valeur de l'éducation et ils comptaient à peine quelques hommes instruits parmi les leurs.

${ }^{20}$ Voir l'histoire de cette congrégation écrite en 1902 par une religieuse non identifiée, Coll. Poirier, CEA, 6.4-14.

21 Pour Le Moniteur Acadien, voir I.-J.-J. Landry à Rameau de SaintPère, 20 mai 1867, CEA, Coll. Rameau, 2.1-8. Pour L'Evangéline, voir Mgr C. O'Brien, Halifax, à Valentin Landry, 15 nov. 1887, CEA, Coll. V.-A. Landry, 7.1-3.

${ }_{22} \mathrm{Mgr}$ T. Connolly, Halifax, à Charles Tupper, s.d., APC, Fonds Tupper, 2: 947-8.

${ }^{23}$ Il y avait 102,000 catholiques romains en Nouvelle-Ecosse dont environ 33,000 étaient d'origine française. Voir Census of Canada, 1871, I: 247 et 332 . 
Moins fortunés, en plus, ils ne pouvaient maintenir des "écoles séparées" sans subside gouvernemental. Déjà ignorés par Mgr Connolly en 1866, ils le furent par les politiciens, les journalistes et les prélats irlandais durant les luttes de 1870 à 1875. En conséquence, leurs intérêts propres furent négligés pendant le débat sur la question des Ecoles du Nouveau-Brunswick. Les catholiques irlandais accueillirent chaleureusement le règlement de la question qui établissait un système apparenté à celui de la Nouvelle-Ecosse. Les Acadiens, eux, demeurèrent mécontents car la loi qui ne prévoyait aucune école française les obligeait à faire instruire leurs enfants dans une langue étrangère. Les écoles, on le craignait, pourraient constituer un autre "agent de destruction nationale" ${ }^{24}$.

Les Acadiens auraient pu faire face à la situation, n'eût été le harcèlement systématique des évêques irlandais. En 1880, un groupe entreprit de faire adopter la St-Jean-Baptiste, fête des Québécois, comme fête nationale des Acadiens. Le choix de ce patron fut controversé car plusieurs, dont le Père Marcel Richard et Pascal Poirier, pensaient qu'il fallait choisir des symboles différents de ceux de la province de Québec, afin de souligner le caractère propre de l'histoire et de la culture acadiennes. La Convention Nationale de 1881 adopta cette dernière position, mais de nombreux Acadiens se montrèrent sceptiques sur l'avenir d'une Acadie isolée du Québec, persistant à croire que "l'élément français (du Canada) ne peut que gagner à serrer ses rangs, à s'entendre, à s'unir" ${ }^{25}$.

D'un point de vue pratique, il aurait été plus sage pour l'Acadie d'adopter saint Jean-Baptiste comme patron national. Le Québec serait ainsi devenu un puissant allié et l'aurait appuyée dans les milieux politiques et ecclésiastiques. Le choix du 24 juin comme fête nationale aurait peut-être forcé les anglophones et les dirigeants du Nouveau-Brunswick à se montrer plus condescendants envers les Acadiens par crainte du Québec. Mais il était évident que la hiérarchie irlandaise s'opposait au choix de la St-Jean-Baptiste et qu'elle aurait refusé de l'accepter ${ }^{26}$. Conscients de ce fait, les Acadiens choisirent la fête de l'Assomption. Dès que les évêques se rendirent compte que cette fête revêtait aussi un caractère nationaliste, ils firent tout en leur pouvoir pour en arrêter le développement.

24 J.-B. Gingras, L'Acadie et Nous (Montréal, 1945), 10.

25 "Discours de L'Hon. P.-A. Landry", dans Le Moniteur acadien, 18 août 1881, 2.

26 Voir P. Poirier à La Patrie (Montréal), 13 déc. 1902, reproduit dans L'Impartial (Tignish, I.P.E.), 15 janv. 1903. 
Il paraît, écrivait l'abbé Biron, que les Evêques Irlandais ne cachent plus leur opposition contre une seconde convention acadienne... l'évêque Rogers était encore plus anti-français qu'on l'avait cru jusqu'ici. La position des prêtres acadiens devient de plus en plus difficile. ${ }^{27}$

Mais la hiérarchie ne pouvait rien contre l'attrait croissant exercé par la fête de l'Assomption. Si. les sentiments ne plient que très rarement devant l'autorité, il en va différemment des institutions. En 1864, Mgr Sweeny, utilisant les immeubles donnés par le Père Lafrance, fonda donc le Collège St-Joseph de Memramcook. C'était une acquisition importante pour la population catholique de la région et, particulièrement, pour les Acadiens qui espéraient que le collège, tel que promis par Mgr Sweeny, bénéficierait principalement à la population acadienne française ${ }^{28}$. Ils croyaient que ce collège favoriserait leur nationalité autant que la foi.

Ce ne fut pas le cas. Le collège devint une institution "bilingue", ce qui signifiait, en fait, que tous les étudiants, acadiens et anglais, parlaient d'abord l'anglais. Un des professeurs du collège, l'abbé Biron, affirma que le bilinguisme à Memramcook était un mythe:

Aujourd'hui l'anglais a une telle supériorité sur le français à Memramcook, où tous les Acadiens parlent l'anglais, tandis qu'on ne peut trouver dix Irlandais sachant le français... on pourrait passer plusieurs journées au milieu des élèves sans entendre un seul mot de français. ${ }^{29}$

Biron était convaincu que l'évêque, le recteur canadien-français et les professeurs irlandais catholiques avaient pris les moyens de rendre le collège inaccessible aux Acadiens car

les Acadiens sont pauvres. Les Anglais, les Américains, et les Irlandais sont plus fortunés et c'est à eux qu'on s'adresse de préférence pour remplir le collège parce qu'ils peuvent mieux que les autres faire prospérer la caisse (de l'institution).$^{30}$

D'abord conçu comme un foyer intellectuel pour les Acadiens de toutes les provinces maritimes, le Collège St-Joseph recruta bien-

27 Abbé E. Biron à Rameau de Saint-Père, 27 juillet 1884, CEA, Coll. Rameau, 2.1-23.

28 Mgr Sweeny, Saint John, à l'abbé Moreau, Montréal, 21 fév. 1863,

Archives du diocèse de Saint John (A.D.S.J.), Coll. Sweeny, lettre no 1850.

29 Abbé Biron à Rameau de Saint-Père, 8 avril 1880, CEA, Coll. Rameau, 2.1-9.

30 Abbé Biron à Rameau de Saint-Père, 17 août 1876, CEA, Coll. Rameau, 2.1-15. 
tôt ses étudiants, dont une minorité seulement était française, dans la région immédiate de Memramcook. ${ }^{31}$

Le mécontentement général des Acadiens incita alors le Père Marcel Richard à fonder, en 1874, un collège rival, à St-Louis de Kent. Acadien dans l'âme, cet ecclésiastique, nationaliste convain$\mathrm{cu}$, ne voyait aucune contradiction entre ses convictions nationalistes et ses devoirs cléricaux. Il voulait construire une institution purement acadienne, et ses efforts, couronnés de succès, furent grandement appréciés des Acadiens qui l'appuyaient. Les étudiants eux-mêmes disaient du Collège St-Louis:

(Nous trouvons ici)

ce que d'autres établissements ne nous offriront pas... une éducation conforme à notre caractère et aux nécessités présentes, un milieu qui nous convenait et nous offrait une grande facilité de rapprochement entre nous par le moyen des élèves déjà nombreux qui accouraient ici de toutes les parties de l'Acadie. ${ }^{32}$

C'était là le but avoué du fondateur. Il avait voulu remédier aux lacunes du Collège St-Joseph, grouper et réunir les étudiants acadiens de toutes les provinces maritimes, leur faire prendre conscience de leur situation et leur inculquer un attachement fervent et sincère à leur race.

L'atmosphère et les objectifs des deux institutions étaient complètement différents. Le Collège St-Joseph desservait la population catholique de Memramcook, et le Collège St-Louis, les Acadiens de toute la région. Le premier voulait préparer ses étudiants à la vie publique, ce qu'il fit parfaitement: de nombreux sénateurs, membres du Parlement, juges, avocats et journalistes sortirent de cette institution. La formation d'une élite acadienne y était secondaire. Le Collège St-Louis, reflétant les priorités du Père Richard, se préoccupait moins de réussite dans le milieu anglo-saxon que de la formation d'une génération de fervents nationalistes acadiens, qui pourraient peut-être, mais en second lieu, accéder à la vie publique. Memramcook préconisait le succès individuel mais dans les limites de la doctrine catholique; St-Louis mettait l'accent sur la responsabilité et le devoir collectifs. La raison d'être de St-Louis était le développement d'une conscience acadienne alors que St-Joseph était simplement un

31 Voir Collège St-Joseph, Annuaires, 1879-1889, CEA. Ces annuaires contiennent la liste des étudiants avec leur provenance. L'auteur appuie ses affirmations sur une étude complète de la région.

32 Les étudiants du Collège St-Louis à Rameau de Saint-Père, juin 1882, CEA, Coll. Rameau, 2.1-21. 
collège catholique fondé sur la foi en la valeur intrinsèque de l'éducation catholique ${ }^{33}$.

La hiérarchie irlandaise du Nouveau-Brunswick n'entrava que rarement l'œuvre du Collège St-Joseph, cette institution n'ayant jamais contesté le statu quo. Cependant, le nationalisme du Père Richard et sa dévotion à la cause acadienne suscitèrent l'antipathie de son évêque, Mgr Rogers de Chatham. Cette antipathie mit en cause l'existence même du collège. Bien que cette institution manquait régulièrement de personnel, Mgr Rogers n'en menaça pas moins fréquemment de nommer certains de ses membres à des cures. Chaque fois que Richard protestait, il était l'objet de sévères réprimandes de la part de son évêque. Leurs différends concernant les politiques du collège s'envenimèrent à tel point qu'en juillet 1882 , Mgr Rogers provoqua une confrontation. Prétendant que "le français y (St-Louis) était trop enseigné" ${ }^{4}$ et que le collège, en conséquence, était devenu "un foyer de discorde" 35 entre Acadiens et Irlandais de la paroisse, il lui retira son appui et le contraint à fermer ses portes. Il est évident que le collège constituait une menace à l'hégémonie irlandaise dans les provinces maritimes parce qu'il produisait une élite instruite et énergique, élite toute pétrie d'un nationalisme acadien intense, tel que prôné par le Père Richard. A moins de se conformer aux vues qu'avait Mgr Rogers sur son diocèse et de s'engager à respecter le statu quo, le collège ne pourrait donc continuer à exister.

D'autre part, si les Acadiens désiraient protéger efficacement leur identité culturelle contre l'envahissement irlandais, ils se devaient d'accéder au sommet de la hiérarchie ecclésiastique. Mais là encore, les obstacles étaient à peu près insurmontables. Tout favorisait l'Irlandais, qui était batailleur de nature. Les Acadiens voyaient leurs efforts entravés par l'attitude traditionnelle de Rome qui s'opposait à l'ingérence des laïcs dans les conflits hiérarchiques. Ils ne pouvaient même pas en appeler à la hiérarchie du Québec, car Rome voyait également d'un mauvais œil l'intervention d'un évêque dans la régie interne d'un autre diocèse. Enfin, les prélats irlandais n'hésitaient pas à recourir

33 Voir supra, n. 31, Fr. M. Gildas, Mgr M.-F. Richard (Moncton, 1940), 41-61; C.-D. Doucet, Mgr Marcel-François Richard, ms. (1973); voir aussi notre thèse de doctorat, The Acadian Renaissance and the Development of Acadian-Canadian Relations, 1864-1912 (Dalhousie University, 1975), 7 .

34 P. Poirier, Les Acadiens - Depuis la Confédération jusqu'à nos jours, ms. (1922), CEA, Coll. Poirier, 6.2-2: 9.

35 Doucet, Mgr M.-F. Richard, c. 8: 9 . 
aux encycliques pour avertir les Acadiens "de ne pas espérer la fin de leurs maux en ce monde mais de plutôt cuirasser leur âme pour supporter les épreuves" ${ }^{36}$ (Léon XIII).

Jusqu'en 1899, les Acadiens nourrirent l'illusion que justice leur serait faite par la hiérarchie irlandaise. Cette année-là, les cinq évêques des provinces maritimes devaient se réunir pour trouver des successeurs aux évêques Rogers et Sweeny, en place depuis 1860. Les Acadiens espéraient la nomination d'un des leurs, mais leurs espérances furent vite déçues car les abbés T. F. Barry et $T$. Casey furent respectivement nommés aux diocèses de Chatham et de Saint John.

Ce dernier affront rendit les Acadiens furieux. Les prêtres acadiens boycottèrent les cérémonies d'intronisation alors que la presse protestait en termes violents et incendiaires. Les Acadiens ne pouvaient s'expliquer l'obstination de la hiérarchie autrement que par le désir d'accaparer le pouvoir. Cependant, le conflit entre Acadiens et Irlandais du Nouveau-Brunswick n'était que le reflet ou le signe avant-coureur d'un conflit beaucoup plus large devant s'étendre à tout le Canada. Les catholiques irlandais et canadiens-français avaient des conceptions différentes des fonctions de l'Eglise dans une société pluraliste. Peu importait l'endroit ou l'occasion, les deux factions se querellaient.

L'écho de ces querelles du Nouveau-Brunswick se fit entendre pour la première fois sur la scène canadienne, en 1910, au Congrès eucharistique de Montréal. Mgr Bourne, archevêque de Westminster, eut l'audace d'exposer les vues des Irlandais à un auditoire en majorité canadien-français. Peu après, l'antagonisme franco-irlandais gagna l'Ontario où Mgr T. F. Fallon, de London, dirigea le mouvement qui avait pour objectif de proscrire le français des écoles de cette province. De là, l'antagonisme s'étendit au Manitoba, à la Saskatchewan et à l'Alberta ${ }^{37}$.

${ }^{36}$ Léon XIII, Encyclique, "De Jesu Christo Redemptore", 1er nov. 1900. Copie adressée à Pierre-A. Landry par Mgr Rogers, CEA, Coll. Landry, 5.5-8. 37 Voir Mason Wade, The French Canadians (Toronto, 1968), II: 608-708, passim; Robert Choquette, "Adélard Langevin et l'érection de l'archidiocèse de Winnipeg", Revue d'histoire de l'Amérique française, 28, no 2 (sept. 1974) : 187-207; Henri Bourassa, Religion. Langue, Nationalité - Discours prononcé par sa Grandeur Mgr Bourne (Montréal, 1910), 19-23; Le Devoir, 14 sept. 1910, p. 1, "Interview Accordé par Mgr Bourne"; pour la question ontarienne, voir la lettre des catholiques du Canada à Mgr Merry Del Val, Rome, 17 juin 1905, en Réponse aux prétendus griefs des catholiques irlandais du Canada contre les catholiques français du même pays, 30 mai 1909, CEA, Coll. Brochures; L'Evangéline, 10 oct. 1910, 3, "Une Entrevue avec Mgr Fallon". 
Les prélats irlandais ne s'opposaient pas aux aspirations canadiennes-françaises ou acadiennes par simple parti pris. Ils considéraient que leurs mobiles d'action étaient tout à fait louables. L'archevêque Bourne, par exemple, se montrait sympathique à la langue française. Il ne désirait pas la voir disparaître complètement, mais il croyait que si le Canada

is to be won for and held to the Catholic Church, this can only be done by making known to the great part of the Canadian people ... the mysteries of our faith through the medium of the English language.

Sa préoccupation n'était pas le statut de la langue française mais "the future of the Church" ${ }^{38}$. Les Irlandais de l'Ontario s'appuyaient sur le même argument lorsqu'ils affirmaient que la "list of converts in a diocese of French Canadian clergy will always be small, as our separated brethen do not find there the intimate friendships which grow from association with Englishspeaking clergy" 39. L'archevêque E. J. McCarthy, d'Halifax, accusait sans détour les Acadiens de mêler à la religion "their narrow feeling of national bigotry" et prétendit que si cette situation était tolérée plus longtemps "the large Protestant element... will cease to enquire into our holy doctrines" ${ }^{40}$.

Il n'y a aucun doute, cependant, que les inquiétudes des évêques concernant l'Eglise s'inspiraient du racisme. Pour se développer et s'étendre, l'Eglise devait se lier à la majorité et non à la minorité linguistique; elle ne devait pas s'identifier à une province (Québec) ou à un peuple dont l'existence même suscitait l'animosité dans de vastes sections de la population canadienne. Les évêques irlandais croyaient que la majorité anglo-protestante ne se méfiait pas de l'Eglise catholique comme telle, mais qu'elle craignait et combattait l'Eglise canadiennefrançaise. De l'avis de ces prélats, la question des Ecoles du Manitoba, par exemple, "would never have occurred, had there been a Bishop of English nationality in the diocese at the time". "Les écoles séparées" soulevaient des objections dans plusieurs provinces, non pas "so much on account of their catholicity as to the domination of French-Canadian episcopacy in an English country" ${ }^{41}$. Un prêtre irlandais de Moncton écrivait que la pré-

38 Bourassa, Religion, Langue, Nationalité, 20-1.

39 Lettre des catholiques irlandais du Canada à Mgr Merry Del Val, Rome, 17 juin 1905.

$40 \mathrm{Mgr}$ E. J. McCarthy à Mgr D. Sbaretti, délégué apostolique au Canada, 17 janv. 1908. Copie à CEA.

41 Lettre des catholiques irlandais du Canada à Mgr Merry Del Val, Rome, 17 juin 1905. 
sence d'un évêque acadien amènerait "a new and most explosive element in the arrangement with ... the Government whereby we are practically assured Catholic schools" ${ }^{42}$.

La hiérarchie catholique, surtout au Nouveau-Brunswick, avait toujours peur de provoquer l'animosité des Anglo-Protestants. Pascal Poirier disait qu'elle avait "plus peur de l'opinion anglaise que de Dieu, du diable." ${ }^{43}$ Depuis 1875 et le règlement de la question des Ecoles du Nouveau-Brunswick, les évêques des provinces maritimes avaient conscience de vivre dans un milieu protestant et faisaient l'impossible pour éviter d'éveiller l'hostilité de la majorité. Au besoin, ils feraient des compromis sur les principes catholiques. Quand le Père Marcel Richard voulut excommunier deux de ses paroissiens, Mgr Rogers l'avisa de n'en rien faire, soulignant "that the reaction against us would ... produce still greater evils than the evile (sic) which we would try to correct... The (Protestants) Ministers and their friends would not fail to retaliate ad nauseum." 44

C'était pour éviter une semblable réaction que la hiérarchie s'opposait à la nomination d'un évêque acadien. Elle savait qu'il existait un grand nombre de protestants fanatiques déjà trop avides d'éveiller le sectarisme religieux, et une telle nomination leur en fournirait l'occasion. En conséquence, les catholiques du Nouveau-Brunswick auraient pu se voir enlever les quelques privilèges acquis. Les évêques étaient d'autant plus fermes dans leur opinion qu'ils croyaient que les Acadiens désiraient plus qu'une juste représentation et aspiraient à une "ultimate domination of the Maritime provinces" 45 .

Ne voulant pas que l'Eglise présente un caractère étranger, les prélats irlandais tentèrent d'éliminer l'influence française des provinces "where it is apparent that English ideas are to predominate" "46. Ils essayèrent de nier, ou tout au moins de cacher l'existence d'un nombre imposant de Canadiens français en dehors du Québec. Malgré une population majoritairement cana-

42 Abbé E. Savage à Mgr E. LeBlanc, Saint John, 18 sept. 1913, dans E. Savage, Thirty Years in Moncton, CEA, "Ville de Moncton". 6.4-1.

43 P. Poirier au Père A. Poirier, 10 mars 1931, CEA, Coll. Poirier,

${ }^{44} \mathrm{Mgr}$ Rogers à M.-F. Richard, 27 janv. 1881, CEA, Coll. Richard, "1881". Le souligné est de l'auteur.

45 Nous appuyons notre affirmation sur une correspondance importante trouvée aux Archives épiscopales d'un des diocèses des Maritimes. Malheureusement, on ne nous a pas permis de citer cette correspondance. 17 juin 1905 .

${ }^{46}$ Lettre des catholiques irlandais du Canada à Mgr Merry Del Val, 
dienne-française, les diocèses de London, d'Alexandria et de Sault Ste-Marie, en Ontario, furent placés sous l'autorité d'évêques irlandais ${ }^{47}$. En 1910, les catholiques irlandais contestèrent et renversèrent la prééminence canadienne-française dans l'archidiocèse d'Ottawa ${ }^{48}$. Au Nouveau-Brunswick, les Acadiens restaient privés d'une juste représentation dans la hiérarchie ecclésiastique.

Aux Acadiens qui les blâmaient de plus en plus de considérer que "they were the Church and the faithful were no part of it" ${ }^{49}$, les prélats irlandais ripostaient avec autant d'agressivité et prétendaient qu'il ne devrait pas y avoir de catholiques irlandais, écossais ou acadiens, mais uniquement des catholiques romains. Ils condamnaient "the national spirit" qui croissait parmi les Acadiens, considérant ce nationalisme comme un "harbinger of much evil to religion"; ils soutenaient aussi que plusieurs leaders acadiens étaient "Frenchmen first and Catholics afterwards" et qu'ils ne tenaient pas compte de la religion d'un individu, à moins qu'il ne fût français ${ }^{50}$. Ils s'opposèrent à une représentation acadienne au sein de l'épiscopat, considérant que cette concession serait
an attempt to divide the seamless garrnent of our Lord... (and consequently) un-Catholic, un-Canadian, un-Christian... I (Rev. Savage) solemnly protest against any attempt to make the Catholic Church a weapon to enforce a purely human ambition (i.e. preservation of the French language) no matter how good or pure, patriotic or sentimental. ${ }^{51}$

Dans le but d'assurer leur complète hégémonie, les évêques irlandais tentèrent de détourner le peuple acadien de sa propre élite en affirmant que Pierre Landry et Pascal Poirier, tous deux catholiques dévots, étaient des ennemis de l'Eglise, des "atheists" dont les œuvres "must in time impress a good innocent people with some sentiments of rebellion against the authority of our holy mother the Church" 52 . Ils parlèrent d'un "evil spirit animating some of these fiery Acadians... (which will) land

${ }^{47}$ L'Evénement, 15 oct. 1909 , "En Garde".

48 Le Devoir, 19 juillet 1910, 1 ; et 24 sept. 1910, 1.

49 P.-A. Landry au Père Gaymor, du Saint, John Freeman, 12 déc. 1901, CEA, Coll. Landry, 5.1-4.

50 Archives épiscopales des provinces maritimes. Voir supra, n. 45.

51 Abbé E. Savage à Mgr E. LeBlanc, 18 sept. 1913, dans Savage, Thirty Years in Moncton, 12.

$52 \mathrm{Mgr}$ T. Casey à Sour Marie-Léonie, 19 oct. 1902, CEA, Coll. Lefebvre, "Mère Léonie, correspondance". 
them where many of the poor people of France are today" ${ }_{53}$. C'est avec force détails que les évêques informèrent Rome que Valentin Landry, l'énergique rédacteur de l'Evangéline, avait épousé devant un ministre protestant la nièce de sa défunte femme ${ }^{54}$. Pendant ce temps, les évêques, rusés comme des politiciens, assuraient les différents délégués apostoliques que les Acadiens étaient "perfectly content" et qu'ils se montraient d'excellents catholiques "although, précisaient-ils, we may say that they were less exposed to contaminating influence being less associated with protestants" 55 . Finalement, ils tentèrent et réussirent partiellement à soustraire aux Acadiens leur appui traditionnel, en muselant par certains honneurs le clergé canadien-français du Nouveau-Brunswick.

Les Acadiens ne purent accepter sans riposte le comportement de leurs évêques car ils étaient convaincus que leur avenir comme entité culturelle distincte reposait sur leur représentation dans la hiérarchie. Ils prenaient conscience que la lutte était inégale, et Pascal Poirier comparait leurs efforts à ceux des "Trois Mousquetaires de Dumas dans un duel contre les 'mignons' du roi" ${ }^{56}$. Néanmoins, les Acadiens parèrent l'attaque des Irlandais avec une dureté impitoyable. Selon leurs dires, c'étaient les Irlandais et non les Anglo-Protestants qui étaient responsables du fanatisme religieux que l'on constatait au Canada. Si, affirmaient les Acadiens, les orangistes du Nouveau-Brunswick étaient "plus civilisés, moins féroces" que ceux d'Ontario, c'est qu'ils n'étaient pas encore venus "en contact avec l'Irlandais catholique" 57. D'après V.-A. Landry, ils menaçaient de "les (Irlandais) chasser à coup de bâtons" 58 , en proclamant: "Il faut que l'Acadie nous soit rendue... il faut que l'Irlandais abandonne notre domaine qu'il terrorise depuis des années. Il faut qu'il tombe et que l'Acadien, encore une fois, gouverne son pays, ses enfants ${ }^{59}$." Certains chefs acadiens suggérèrent même, quoique discrètement, d'en venir au boycottage des dignitaires et des

${ }^{53}$ Mgr T. Casey au Père Cormier, 4 nov. 1905, CEA, Coll. Landry,

54 Archives épiscopales des provinces maritimes. Voir supra, n. 45. à CEA.

55 Mgr E. J. McCarthy, Halifax, à Mgr D. Sbaretti, 17 janv. 1908. Copie

56 P. Poirier au Père A. Poirier, 10 mars 1931, CEA, Coll. Poirier, 6.4-1.

67 V.-A. Landry, manuscrit sans titre, mai 1911, CEA, Coll. V.-A. Landry, 7.2-10.

58 V.-A. Landry à Mgr D. Sbaretti, 25 juill. 1908, CEA, Coll. V.-A. Landry, 7.1-17. Le souligné est de l'auteur. 7.1-18.

59. V.-A. Landry à P. Poirier, 14 mars 1909, CEA, Coll. V.-A. Landry, 
prêtres peu sympathiques à leur cause ${ }^{60}$. Rome s'allarma et s'émut de ces luttes fratricides surtout quand elles donnèrent lieu aux innombrables pétitions acadiennes et aux deux pèlerinages du Père Richard à Rome. En 1912, les Acadiens avaient convaincu les autorités romaines du bien-fondé de leurs revendications, et le premier évêque acadien, Mgr Edouard LeBlanc, fut nommé au diocèse de Saint John.

Le comportement des Acadiens tout au long de cette lutte traduisait mal leur vrai caractère national. Ce peuple, peut-être en raison de son histoire, était fondamentalement passif et patient, se faisant une obligation de tenir compte du type de société dans laquelle il se trouvait et d'évoluer avec prudence vers le statut d'égalité:

Ce qu'il est impossible d'obtenir en bloc, et tout d'un coup ... . contentons-nous de tâcher de l'obtenir graduellement, un lambeau ici, un lambeau là, ce sera toujours autant, et de la sorte nous finirons par avoir la majeure partie de ce qu'il est inutile de chercher autrement. ${ }^{61}$

Mais si les Acadiens adoptaient une telle attitude face au monde anglo-protestant des affaires, de la politique et du gouvernement, là où ils constituaient une très faible minorité, il n'en était pas ainsi à l'intérieur de leur propre Eglise. Leur nombre et leur loyauté auraient dû leur mériter dans cette Eglise une représentation adéquate et un minimum de justice de la part de leurs supérieurs cléricaux.

Il est évident que les Acadiens et les Irlandais n'auraient pas dû se laisser aller à cette guerre religieuse qui s'expliquait, en grande partie, par l'émotion et la mesquinerie et qui portait préjudice aux véritables intérêts des deux races. John Costigan, ministre de la Couronne dans le cabinet MacDonald et plus tard sénateur, fut l'un des seuls à estimer que "if French Catholics and Irish Catholics were united... no government would be powerful enough to put them down" ${ }^{62}$. Cependant les divergences s'expliquaient par de nombreuses raisons: ambitions personnelles, intérêt réel pour l'avenir de l'Eglise, conceptions différentes du rôle de cette institution dans une société laïque. La raison fondamentale, cependant, tenait dans ce fait: la hiérarchie irlandaise qui se protégeait elle-même ne pouvait tout

60 L'Evangéline, 23 sept. 1909, 2.

61 Le Moniteur Acadien, 16 déc. 1880.

62 Canada, Debates of the Senate, 5ème Session, 12ème Parlement, 18 mars 1915, 126. 
simplement pas comprendre que, pour l'Acadien comme pour le Canadien français, race et religion étaient absolument inséparables.

Décidés à préserver leur identité ethnique, les Acadiens ne pouvaient plus, en 1900, accepter l'ostracisme avilissant et systématique dont ils étaient l'objet de la part des Irlandais. L'Eglise catholique et son clergé constituaient encore des éléments essentiels de la société canadienne-française ou acadienne et, si l'Eglise ne se compromettait pas pour la sauvegarde de la race francaise au Canada, cette cause serait perdue. L'Irlandais justifiait sa volonté de domination en prétextant un éventuel rapprochement avec les protestants, justification fort douteuse dans les circonstances. Les Acadiens, sinon des Canadiens français, avaient prouvé, eux, qu'ils pouvaient collaborer avec leurs voisins anglo-saxons et mériter leur respect. Mais comme la domination irlandaise prolongée aurait annihilé leurs efforts de survie et signé leur arrêt de mort, ils avaient lutté farouchement pour être représentés au sein de la hiérarchie. Une fois ce but atteint, ils étendirent leur emprise, ainsi que l'avaient craint les prélats irlandais, sur l'épiscopat catholique du NouveauBrunswick. Le résultat, comme l'avaient encore craint les Irlandais, ne marqua pas la fin des divisions raciales faites au nom du catholicisme, mais leur perpétuation. Pour les Acadiens, cependant, ce conflit racial assura, accrut et scella leur adhésion à la foi catholique romaine.

Traduction d'AINDRÉE DÉSILETS

Université de Sherbrooke 